\title{
The role of the Roper in QCD
}

\author{
S R Beane ${ }^{1,2,3}$ and U van Kolck ${ }^{4,5}$ \\ ${ }^{1}$ Institute for Nuclear Theory, University of Washington, Seattle, WA 98195-1560, USA \\ 2 Department of Physics, University of New Hampshire, Durham, NH 03824-3568, USA \\ 3 Jefferson Laboratory, 12000 Jefferson Avenue, Newport News, VA 23606, USA \\ ${ }^{4}$ Department of Physics, University of Arizona, Tucson, AZ 85721, USA \\ 5 RIKEN-BNL Research Center, Brookhaven National Laboratory, Upton, NY 11973, USA
}

Received 13 January 2005

Published 24 June 2005

Online at stacks.iop.org/JPhysG/31/921

\begin{abstract}
We show that existing data suggest a simple scenario in which the nucleon and the $\Delta$ and Roper resonances act as chiral partners in a reducible representation of the full QCD chiral symmetry group. We discuss the peculiar interpretation of this scenario using spin-flavour symmetries of the naive constituent quark model, as well as the consistency of the scenario with large- $N_{c}$ expectations.
\end{abstract}

\section{Introduction}

Understanding of the pattern of baryon masses and couplings from QCD remains an open challenge for theorists. The need for theoretical progress is at least partly driven by the prospect of new experimental results. The CLAS collaboration at JLab expects to significantly improve knowledge of excited baryon masses and decays [1], as does ELSA at Bonn [2], MAMI at Mainz [3] and BES at BEPC [4], among others. Complementary efforts are well underway to compute properties of excited baryons using lattice QCD $[5,6]$. Progress has also been made in analysing the excited baryons in the $1 / N_{c}$ expansion [7, 8]. In the large- $N_{c}$ limit, the light baryons fall into representations of a contracted $S U\left(2 N_{f}\right)$ spin-flavour symmetry where $N_{f}$ is the number of active flavours [9]. This symmetry is highly predictive; it determines inter alia ratios of axial-vector couplings and magnetic moments, and gives rigorous justification to several aspects of the naive constituent quark model (NCQM).

One particularly interesting unresolved issue in baryon spectroscopy is that of the role of the Roper resonance, $N(1440)$ or $N^{\prime}$. In the most naive interpretation, $N^{\prime}$ is a three-quark radial excitation of the nucleon with the same spin-parity quantum numbers. However, this interpretation has been questioned for several reasons. First, the calculated mass of $N^{\prime}$ would appear to be too high in quark models [10]. (Models with explicit pion degrees of freedom do not seem to suffer from this drawback. See, for instance, [11, 12].) Second, several quenched lattice QCD calculations find a spectrum inverted with respect to experiment, with $N^{\prime}$ heavier than the first excited state with opposite parity [5]. More recent studies [6], which implement careful analyses of statistical and systematic errors, suggest that the observed order 
is restored as the pion mass decreases, pointing to an important role for chiral symmetry in the spectrum. An alternative interpretation is that the Roper is a hybrid state; that is, it couples predominantly to QCD currents with some gluonic contribution [13, 14], or is generated by the meson-baryon dynamics [15]. Recently, the consequences of spin-flavour $S U$ (6) for the Roper multiplet have been worked out in the large- $N_{c}$ expansion, assuming that the Roper is in a 56-dimensional representation of spin-flavour $S U(6)$ (the 20-dimensional representation of spin-flavour $S U(4)$ ) [8]. Several predictions have been made which will be tested experimentally at JLab and other experimental facilities.

In this paper we consider consequences of chiral symmetry for the low-lying light baryons. When chiral constraints on the baryons are discussed it is almost always in the context of the limiting scenario of chiral-symmetry restoration ${ }^{6}$. By contrast, we will consider consequences of the full QCD chiral symmetry group in the broken phase for the light baryons. An important message that this paper hopes to convey is that there is no need to summon extreme conditions in order to find consequences of chiral symmetry in the broken chiral symmetry phase. The formalism necessary to extract the consequences of chiral symmetry for baryons was developed by Weinberg and others many years ago [18-24]. We find it surprising that this ancient wisdom is not widely known. Thus while much of what we present in this paper is not new, we feel that the time is ripe for a reassessment of these powerful methods.

We first motivate our discussion by performing an updated analysis of the well-known Adler-Weisberger sum rule for pion-nucleon $(\pi N)$ scattering [25]. We then show that in the resonance-saturation approximation, the Adler-Weisberger sum rule can be derived directly from the chiral $S U(2) \times S U(2)$ algebra through a simple application of the Wigner-Eckart theorem. Not surprisingly, the most powerful way of deriving consequences of the chiral algebra is by using the group representation theory: that is, by requiring that the baryons transform as sums of allowed irreducible representations. Now, naively one might expect that these representations are infinite dimensional and that consequently chiral symmetry gives very little predictive power in the low-energy theory. However, as we will show, data suggest otherwise. In particular, we will make the case that the ground-state chiral multiplet is composed of the nucleon, $N(940)$, the $\Delta$ resonance, $\Delta(1232)$, and the Roper resonance, $N(1440)$, which fall into a reducible $\left(0, \frac{1}{2}\right) \oplus\left(\frac{1}{2}, 1\right)$ representation of $S U(2) \times S U(2)$ with approximately maximal mixing. This representation offers a compelling interpretation of the nucleon axial couplings and of the special role of the Roper resonance in QCD.

We offer an interpretation of our results in the context of the NCQM. We find that our proposed chiral representation is equivalent to placing the nucleon and the $\Delta$ and Roper resonances in a reducible $\mathbf{4} \oplus \mathbf{2 0}$ representation of spin-flavour $S U(4)$. While this scenario is consistent with large- $N_{c}$ QCD, the naive large- $N_{c}$ counting is badly violated by experiment. Moreover, we find that our results are not consistent with placing the Roper in a $\mathbf{2 0}$-dimensional representation of spin-flavour $S U(4)$, as is usually assumed; rather, consistency of our results with large- $N_{c}$ would require that the Roper be in the fundamental representation of $S U(4)$ in the large- $N_{c}$ limit.

\section{The Adler-Weisberger sum rule}

\subsection{The dispersion relation}

Consider the renowned Adler-Weisberger sum rule [25],

$$
g_{A}^{2}=1-\frac{2 f_{\pi}^{2}}{\pi} \int_{0}^{\infty} \frac{\mathrm{d} v}{v}\left[\sigma^{\pi^{-} p}(v)-\sigma^{\pi^{+} p}(v)\right] .
$$

${ }^{6}$ For several recent attempts, see [16, 17]. 
Table 1. Resonances which contribute to the Adler-Weisberger sum rule for $\pi N$ scattering. We have used PDG central values and estimates. We emphasize that there is substantial uncertainty in these values. Only established resonances $(\star \star \star$ and $\star \star \star \star)$ have been tabulated.

\begin{tabular}{llllll}
\hline & $\mathcal{R}$ & $\mathcal{I}_{\mathcal{R}}$ & & $\mathcal{R}$ & $\mathcal{I}_{\mathcal{R}}$ \\
\hline$P_{11}\left(\frac{1}{2}^{+}\right)$ & $N(940)$ & - & $P_{11}\left(\frac{1}{2}^{+}\right)$ & $N(1710)$ & 0.01 \\
$P_{33}\left(\frac{3}{2}^{+}\right)$ & $\Delta(1232)$ & 1.02 & $P_{13}\left(\frac{3}{2}^{+}\right)$ & $N(1720)$ & 0.02 \\
$P_{11}\left(\frac{1}{2}^{+}\right)$ & $N(1440)$ & 0.23 & $F_{35}\left(\frac{5}{2}^{+}\right)$ & $\Delta(1905)$ & 0.02 \\
$D_{13}\left(\frac{3}{2}^{-}\right)$ & $N(1520)$ & 0.09 & $P_{31}\left(\frac{1}{2}^{+}\right)$ & $\Delta(1910)$ & 0.01 \\
$S_{11}\left(\frac{1}{2}^{-}\right)$ & $N(1535)$ & 0.04 & $P_{33}\left(\frac{3}{2}^{+}\right)$ & $\Delta(1920)$ & 0.01 \\
$P_{33}\left(\frac{3}{2}^{+}\right)$ & $\Delta(1600)$ & 0.06 & $D_{35}\left(\frac{5}{2}^{-}\right)$ & $\Delta(1930)$ & 0.03 \\
$S_{31}\left(\frac{1}{2}^{-}\right)$ & $\Delta(1620)$ & 0.02 & $F_{37}\left(\frac{7}{2}^{+}\right)$ & $\Delta(1950)$ & 0.08 \\
$S_{11}\left(\frac{1}{2}^{-}\right)$ & $N(1650)$ & 0.04 & $G_{17}\left(\frac{7}{2}^{-}\right)$ & $N(2190)$ & 0.03 \\
$D_{15}\left(\frac{5}{2}^{-}\right)$ & $N(1675)$ & 0.08 & $H_{19}\left(\frac{9}{2}^{+}\right)$ & $N(2220)$ & 0.03 \\
$F_{15}\left(\frac{5}{2}^{+}\right)$ & $N(1680)$ & 0.10 & $G_{19}\left(\frac{9}{2}^{-}\right)$ & $N(2250)$ & 0.02 \\
$D_{13}\left(\frac{3}{2}^{-}\right)$ & $N(1700)$ & 0.01 & $H_{3,11}\left(\frac{11}{2}^{+}\right)$ & $\Delta(2420)$ & 0.02 \\
$D_{33}\left(\frac{3}{2}^{-}\right)$ & $\Delta(1700)$ & 0.03 & $I_{1,11}\left(\frac{11}{2}^{-}\right)$ & $N(2600)$ & 0.02 \\
\hline
\end{tabular}

Here $g_{A}$ is the nucleon axial-vector coupling, $f_{\pi} \simeq 93 \mathrm{MeV}$ is the pion decay constant and $\sigma^{\pi^{ \pm} p}$ is the total cross-section for charged pion scattering on a proton. Recall that this sum rule for the $\pi N$ scattering amplitude follows from two inputs: (i) a chiral symmetry lowenergy theorem and (ii) the assumption that the forward $\pi N$ amplitude with isospin, $I=1$, in the $t$-channel satisfies an unsubtracted dispersion relation. Saturating the sum rule with $N$ $(I=1 / 2)$ and $\Delta(I=3 / 2)$ resonances gives

$$
g_{A}^{2}=1-\sum_{N} \mathcal{I}_{N}+\sum_{\Delta} \mathcal{I}_{\Delta}+\text { continuum },
$$

where the $\mathcal{I}_{\mathcal{R}}$ are related to experimental widths by

$$
\mathcal{I}_{\mathcal{R}}=\frac{64 \pi f_{\pi}^{2} M_{\mathcal{R}}^{3}}{3\left(M_{\mathcal{R}}^{2}-M_{N}^{2}\right)^{3}}\left(S_{\mathcal{R}}+\frac{1}{2}\right) \Gamma^{\mathrm{TOT}}(\mathcal{R} \rightarrow N \pi),
$$

and $S_{\mathcal{R}}$ is the spin of the resonance $\mathcal{R}$.

We can now go to the particle data group (PDG) [26] and compute the contribution of each $N$ and $\Delta$ state to the sum rule (see table 1 ). We include only established resonances $(\star \star \star$ and $\star \star \star \star)$, using PDG central values and estimates. We find $\sum \mathcal{I}_{N}=0.72$ and $\sum \mathcal{I}_{\Delta}=1.3$. Neglecting the continuum contribution (we will return to this point below), we then obtain $g_{A}=1.26$, to be compared to the experimental value of $1.2670 \pm 0.0035$ [26]. This is truly remarkable agreement. There are several important things to note from table 1 . First, there is a cancellation between the $N$-and $\Delta$-type contributions, which enter with opposite sign. Second, $\Delta(1232)$ and $N(1440)$ dominate the sum rule. Axial transitions of the excited baryons to the ground-state nucleon are small compared to the dominant transitions. For instance, saturating the sum rule with these two states alone gives $g_{A}=1.34$.

Given the uncertainties in the resonance masses and axial couplings, and the neglect of the continuum contribution, such remarkable agreement must to some degree be fortuitous. Given the success of the sum rule one might ask: what precisely is the sum rule testing about QCD? What is the significance of the assumption about the asymptotic behaviour of the forward $\pi N$ 
scattering amplitude? Why do $\Delta(1232)$ and $N(1440)$ seem to have special status in saturating the sum rule? In order to answer these questions we will rephrase the discussion of the sum rule entirely in the language of chiral symmetry.

\subsection{The symmetry interpretation}

In the limit of vanishing up and down quark masses, QCD has an $S U(2)_{L} \times S U(2)_{R}$ invariance. We can write the chiral algebra as

$\left[\mathcal{Q}_{a}^{A}, \mathcal{Q}_{b}^{A}\right]=\mathrm{i} \epsilon_{a b c} T_{c}, \quad\left[T_{a}, \mathcal{Q}_{b}^{A}\right]=\mathrm{i} \epsilon_{a b c} \mathcal{Q}_{c}^{A}, \quad\left[T_{a}, T_{b}\right]=\mathrm{i} \epsilon_{a b c} T_{c}$,

where $T^{a}$ are $S U(2)_{V}$ generators and $\mathcal{Q}_{a}^{A}$ are the remaining axial generators. We define the axial-vector coupling matrix,

$$
\left\langle\beta, \lambda\left|\mathcal{Q}_{a}^{A}\right| \alpha, \lambda^{\prime}\right\rangle=\left[X_{a}^{\lambda}\right]_{\beta \alpha} \delta_{\lambda \lambda^{\prime}},
$$

where $|\alpha, \lambda\rangle$ is a baryon state of definite helicity $\lambda$. Note the Kronecker delta on the right-hand side of this equation. This implies that we are defining $X_{a}^{\lambda}$ in a helicity-conserving Lorentz frame [20]. A frame in which all momenta are collinear is such a frame, as is the infinitemomentum frame. We are of course free to choose any frame. However, helicity-conserving frames are special because in these frames chirality conservation becomes the same as helicity conservation.

The physical consequences of the full chiral symmetry group can now be found by taking matrix elements of the $S U(2) \times S U(2)$ algebra and using the Wigner-Eckart theorem to express the algebra as a set of equations for reduced matrix elements [20]. Taking matrix elements of the $S U(2) \times S U(2)$ algebra of equation (4) and inserting a complete set of states gives

$$
\left[X_{a}^{\lambda}, X_{b}^{\lambda}\right]_{\beta \alpha}=\mathrm{i} \epsilon_{a b c}\left[T_{c}\right]_{\beta \alpha} .
$$

This is a (generalized) Adler-Weisberger sum rule. An important comment is in order here. One might suspect that the vacuum should contribute in the sum over states and that the axial generator acting on the vacuum will generate quark-antiquark pairs, thus destroying the group algebraic structure. The advantage of working in a helicity-conserving frame is that vacuum does not contribute in the sum over states; i.e. $\mathcal{Q}_{a}^{A}|0\rangle=0^{7}$. The chiral symmetry is, however, broken spontaneously: although $X_{a}^{\lambda}$ satisfies the chiral algebra, it does not commute with the baryon mass-squared matrix and is therefore not a symmetry generator. Hence in helicity-conserving frames, all evidence of symmetry breaking is in the Hamiltonian and not in the states. Using the Wigner-Eckart theorem, we can write

$$
\left\langle I_{\beta}, m_{\beta}\left|X_{(m)}^{\lambda}\right| I_{\alpha}, m_{\alpha}\right\rangle=C_{I_{\alpha} 1}\left(I_{\beta}, m_{\beta} ; m_{\alpha}, m\right) X^{\lambda}(\beta, \alpha),
$$

where the $C$ are Clebsch-Gordan coefficients and the $X^{\lambda}(\beta, \alpha)$ are reduced matrix elements [20]. Taking matrix elements of equation (6) between states of definite isospin and inserting a complete set of states then gives a set of coupled equations for the reduced matrix elements $[20,24]$. It is easy to show that for $I_{\alpha}=I_{\beta}=\frac{1}{2}$, equation (6) yields

$$
\sum_{\gamma=N} X^{\lambda}(\beta, \gamma) X^{\lambda \dagger}(\gamma, \alpha)-\sum_{\gamma=\Delta} X^{\lambda}(\beta, \gamma) X^{\lambda \dagger}(\gamma, \alpha)=\frac{3}{4} \delta_{\alpha \beta} .
$$

The coupling of most interest here is

$$
g_{A}=\sqrt{2}\left\langle p\left|X_{(+)}^{ \pm \frac{1}{2}}\right| n\right\rangle=\sqrt{\frac{4}{3}} X^{ \pm \frac{1}{2}}(p, n),
$$

7 Note that the derivation of equation (6) given in [20] avoids any discussion of the QCD vacuum. 
where $X_{( \pm)} \equiv \mp \frac{1}{\sqrt{2}}\left(X_{1} \pm \mathrm{i} X_{2}\right)$ and we are ignoring overall phases. From equations (8) and (9), with $\alpha=\beta=N$ and $\gamma=\mathcal{R}$, it immediately follows that

$g_{A}^{2}=1-\frac{4}{3} \sum_{\mathcal{R}=N} X^{\frac{1}{2}}(N, \mathcal{R}) X^{\frac{1}{2} \dagger}(\mathcal{R}, N)+\frac{4}{3} \sum_{\mathcal{R}=\Delta} X^{\frac{1}{2}}(N, \mathcal{R}) X^{\frac{1}{2} \dagger}(\mathcal{R}, N)$,

which is none other than equation (2) when we identify

$$
\mathcal{I}_{\mathcal{R}}=\frac{4}{3} X^{\frac{1}{2}}(N, \mathcal{R}) X^{\frac{1}{2} \dagger}(\mathcal{R}, N) .
$$

We have now derived the Adler-Weisberger sum rule in two ways. This is not surprising: in [20], Weinberg proved that the assumption that the forward $\pi N$ amplitude with $I=1$ in the $t$-channel satisfies an unsubtracted dispersion relation is equivalent to the algebraic statement of equation (6) in helicity-conserving frames. In his original derivation, Weinberg used Regge pole theory to derive the asymptotic constraint, which then led to equation (6).

Note that, as a bonus, we can derive from equation (8) relations for other couplings of interest. These couplings involve, beside the ground-state nucleon $N$, also the lowest excitations such as the $\Delta(1232)$ and the $N(1440)$. For example, we can define

$$
\begin{aligned}
& g_{A}^{\prime}=\sqrt{2}\left\langle p\left|X_{(+)}^{ \pm \frac{1}{2}}\right| n^{\prime}\right\rangle=\sqrt{\frac{4}{3}} X^{ \pm \frac{1}{2}}\left(p, n^{\prime}\right), \\
& g_{A}^{\prime \prime}=\sqrt{2}\left\langle p^{\prime}\left|X_{(+)}^{ \pm \frac{1}{2}}\right| n^{\prime}\right\rangle=\sqrt{\frac{4}{3}} X^{ \pm \frac{1}{2}}\left(p^{\prime}, n^{\prime}\right), \\
& \mathcal{C}_{\Delta N}=\sqrt{3}\left\langle p\left|X_{(+)}^{ \pm \frac{1}{2}}\right| \Delta^{++}\right\rangle=\sqrt{3} X^{ \pm \frac{1}{2}}\left(p, \Delta^{++}\right), \\
& \mathcal{C}_{\Delta N^{\prime}}=\sqrt{3}\left\langle p^{\prime}\left|X_{(+)}^{ \pm \frac{1}{2}}\right| \Delta^{++}\right\rangle=\sqrt{3} X^{ \pm \frac{1}{2}}\left(p^{\prime}, \Delta^{++}\right),
\end{aligned}
$$

where $n^{\prime}\left(p^{\prime}\right)$ is the neutral (charged) member of the $N^{\prime}$ isodoublet. In the simple scheme in which we saturate the sum rule with $\Delta(1232)$ and the $N(1440)$, we find

$$
g_{A}^{2}+g_{A}^{\prime 2}=1+\frac{4}{9} \mathcal{C}_{\Delta N}^{2} .
$$

One can easily find additional relations among these parameters by taking appropriate matrix elements of equation (6) and constructing the Adler-Weisberger sum rules for $\pi \Delta$ and $\pi N^{\prime}$ scattering. However, as we will see next, it is far more general and practical to work directly with the representations of the chiral symmetry group.

\section{The chiral representation theory}

\subsection{Axial-vector couplings}

Any consequence of the chiral algebra can be obtained from the group representation theory. We will do so now, as it proves to be a much more powerful means of extracting consequences of chiral symmetry. The massless flavours of the underlying QCD Lagrangian transform as $\left(\frac{1}{2}, 0\right)$ and $\left(0, \frac{1}{2}\right)$ with respect to $S U(2)_{L} \times S U(2)_{R}$. Baryons made of light flavours are in general reducible sums of any number of irreducible representations, constrained only by isospin. For $N_{c}=3$, the allowed irreducible representations are $\left(\frac{1}{2}, 0\right),\left(0, \frac{1}{2}\right),\left(\frac{3}{2}, 0\right),\left(0, \frac{3}{2}\right),\left(1, \frac{1}{2}\right)$ and $\left(\frac{1}{2}, 1\right) .8$ Since $\left(\frac{1}{2}, 1\right)$ contains isospin $\frac{1}{2}$ and $\frac{3}{2}$, we will differentiate the isospin states by a subscript. The most general nucleon and $\Delta$ states with $\lambda=\frac{1}{2}$ can be written as

$$
\left|N, \frac{1}{2}\right\rangle=\sum_{l} a_{l}\left|\frac{1}{2}, 0\right\rangle^{(l)}+\sum_{k} b_{k}\left|0, \frac{1}{2}\right\rangle^{(k)}+\sum_{m} c_{m}\left|\frac{1}{2}, 1\right\rangle_{\frac{1}{2}}^{(m)}+\sum_{n} d_{n}\left|1, \frac{1}{2}\right|_{\frac{1}{2}}^{(n)},
$$

\footnotetext{
8 These representations correspond to the dimensionalities, $(\mathbf{2}, \mathbf{1}),(\mathbf{1}, \mathbf{2}),(\mathbf{4}, \mathbf{1}),(\mathbf{1}, \mathbf{4}),(\mathbf{3}, \mathbf{2})$ and $(\mathbf{2}, \mathbf{3})$, respectively.
} 
$\left|\Delta, \frac{1}{2}\right\rangle=\sum_{l} e_{l}\left|\frac{3}{2}, 0\right\rangle^{(l)}+\sum_{k} f_{k}\left|0, \frac{3}{2}\right\rangle^{(k)}+\sum_{m} g_{m}\left|\frac{1}{2}, 1\right|_{\frac{3}{2}}^{(m)}+\sum_{n} h_{n}\left|1, \frac{1}{2}\right\rangle_{\frac{3}{2}}^{(n)}$,

where $a_{l}, \ldots, h_{n}$ are a priori unknown mixing parameters. Parity conservation implies that the $\lambda=-\frac{1}{2}$ representation is obtained from equation (14) by interchanging $S U(2)_{L}$ and $S U(2)_{R}$ representations; i.e. $|\mathcal{A}, \mathcal{B}\rangle \rightarrow|\mathcal{B}, \mathcal{A}\rangle$ where $\mathcal{A} \in S U(2)_{L}$ and $\mathcal{B} \in S U(2)_{R}$ [20]. It is important to realize that the chiral multiplet structure in the broken chiral symmetry phase has nothing to do with parity doubling. We define the axial-vector couplings of the $\lambda=\frac{1}{2}$ nucleon as

$$
\begin{aligned}
& \left\langle N, \frac{1}{2}\left|\mathcal{Q}_{a}^{A}\right| N, \frac{1}{2}\right\rangle=g_{A}^{(1 / 2)}\left[T_{a}\right]_{\frac{1}{2} \frac{1}{2}}, \\
& \left\langle N, \frac{1}{2}\left|\mathcal{Q}_{a}^{A}\right| \Delta, \frac{1}{2}\right\rangle=\sqrt{\frac{2}{3}} \mathcal{C}_{\Delta N}^{(1 / 2)}\left[T_{a}\right]_{\frac{1}{2} \frac{3}{2}},
\end{aligned}
$$

where $\mathcal{Q}_{a}^{A}$ is an $S U(2)_{A}$ generator and $\left[T_{a}\right]_{\alpha \beta}$ is the matrix element of the isospin operator between baryon states of isospin $\alpha$ and $\beta$. Note that the axial-vector coupling carries a helicity superscript.

The action of the QCD generators on the states of definite chirality is given by

$$
\begin{aligned}
& \left\langle 0, \frac{1}{2}\left|\mathcal{Q}_{a}^{A}\right| 0, \frac{1}{2}\right\rangle=-\left\langle\frac{1}{2}, 0\left|\mathcal{Q}_{a}^{A}\right| \frac{1}{2}, 0\right\rangle=-\left[T_{a}\right]_{\frac{1}{2} \frac{1}{2}}, \\
& { }_{\frac{1}{2}}\left\langle\frac{1}{2}, 1\left|\mathcal{Q}_{a}^{A}\right| \frac{1}{2}, 1\right\rangle_{\frac{1}{2}}=-\frac{1}{2}\left\langle 1, \frac{1}{2}\left|\mathcal{Q}_{a}^{A}\right| 1, \frac{1}{2}\right\rangle_{\frac{1}{2}}=-\frac{5}{3}\left[T_{a}\right]_{\frac{1}{2} \frac{1}{2}}, \\
& { }_{\frac{1}{2}}\left\langle\frac{1}{2}, 1\left|\mathcal{Q}_{a}^{A}\right| \frac{1}{2}, 1\right\rangle_{\frac{3}{2}}=-\frac{1}{2}\left\langle 1, \frac{1}{2}\left|\mathcal{Q}_{a}^{A}\right| 1, \frac{1}{2}\right\rangle_{\frac{3}{2}}=2 \sqrt{\frac{2}{3}}\left[T_{a}\right]_{\frac{1}{2} \frac{3}{2}} .
\end{aligned}
$$

Using equation (14) we can now find expressions for the axial couplings in terms of the coefficients $a_{l}, \ldots, h_{n}$. For example, putting equation (14a) into equation (15) and using equation (16), we find for $\lambda=+\frac{1}{2}$

$$
g_{A}^{(1 / 2)}=\frac{\sum_{l}\left|a_{l}\right|^{2}-\sum_{k}\left|b_{k}\right|^{2}-\frac{5}{3}\left(\sum_{m}\left|c_{m}\right|^{2}-\sum_{n}\left|d_{n}\right|^{2}\right)}{\sum_{l}\left|a_{l}\right|^{2}+\sum_{k}\left|b_{k}\right|^{2}+\sum_{m}\left|c_{m}\right|^{2}+\sum_{n}\left|d_{n}\right|^{2}},
$$

with the opposite sign holding for $g_{A}^{(-1 / 2)}$. Similar relations can be derived for other axial couplings. We see from this expression that the baryon axial couplings are completely determined by the angles that mix the states of definite chirality.

\subsection{The mass-squared matrix}

In order to see the effects of spontaneous symmetry breaking, we must consider the baryon mass-squared matrix ${ }^{9}$. How does the mass-squared matrix transform with respect to $S U(2)_{L} \times S U(2)_{R}$ ? In principle the mass-squared matrix, $\hat{M}^{2}$, can transform as a singlet plus any non-trivial representation(s) of the chiral group. Here we will assume

$$
\hat{M}^{2}=\hat{M}_{0}^{2}+\hat{M}_{\bar{q} q}^{2}
$$

where $\hat{M}_{0}^{2} \in(0,0)$ and $\hat{M}_{\bar{q} q}^{2} \in\left(\frac{1}{2}, \frac{1}{2}\right)$. In [20], Weinberg showed that the assumption that the forward $\pi N$ amplitude with $I=2$ in the $t$-channel satisfies a superconvergence relation

9 Hadron resonances are, of course, unstable with respect to the strong interaction, which is reflected in non-vanishing decay widths. Here we assume that QCD can be represented by an effective hadronic theory where resonances are represented by explicit fields with real mass terms, and widths are generated by loop diagrams. A detailed discussion of the role of resonances in effective theories can be found in [27], and references therein. 
is equivalent to the algebraic statement of equation (18) in helicity-conserving frames. All chiral symmetry breaking in the baryon sector is then contained in matrix elements of the form $\left\langle 0, \frac{1}{2}\left|\hat{M}_{\bar{q} q}^{2}\right| \frac{1}{2}, 1\right\rangle$ and $\left\langle 0, \frac{1}{2}\left|\hat{M}_{\bar{q} q}^{2}\right| \frac{1}{2}, 0\right\rangle$. The mass of a baryon $B$ is defined as

$$
M_{B}^{2}=\left\langle B, \frac{1}{2}\left|\hat{M}^{2}\right| B, \frac{1}{2}\right\rangle .
$$

If one assumes that there is no inelastic diffractive scattering [20], there is an additional superconvergence relation which can be expressed algebraically as

$$
\left[\hat{M}_{0}^{2}, \hat{M}_{\bar{q} q}^{2}\right]=0 \text {. }
$$

This rather peculiar commutator constrains the mixing angles in reducible representations, as we will see below.

\section{Chiral representations}

In principle the baryon representations can be infinite dimensional. If this were the case, it would be unlikely that chiral symmetry would have any predictive power for the baryons. Fortunately, the phenomenological analysis of the Adler-Weisberger relation presented in section 2 suggests that the baryon representations are small. In this section we will consider the consequences of the simplest baryon representations. We will be primarily concerned with the pion transitions of the ground-state nucleon. Therefore, it is sufficient to consider the $\lambda= \pm \frac{1}{2}$ sector, although other helicities can be considered as well. We will ignore the overall phases of the axial couplings. They can easily be found using equations (15) and (16).

\subsection{The sigma model}

The simplest chiral representation places the nucleon helicity states in the $\left(\frac{1}{2}, 0\right)$ or $\left(0, \frac{1}{2}\right)$ representations. This corresponds to $a_{l}=c_{m}=d_{n}=0$ and $b_{k}=\delta_{k 1}$ in equation (14a):

$$
\left|N, \frac{1}{2}\right\rangle=\left|0, \frac{1}{2}\right\rangle, \quad\left|N,-\frac{1}{2}\right\rangle=\left|\frac{1}{2}, 0\right\rangle .
$$

From equation (17) we find immediately that $g_{A}=1$. This prediction follows exclusively from chiral symmetry and the assumed representation. This result is familiar from the linear sigma model where there is one nucleon field, $N=N_{L}+N_{R}$, with $N_{L} \in\left(\frac{1}{2}, 0\right)$ and $N_{R} \in\left(0, \frac{1}{2}\right)$, and one finds $g_{A}=1$. Here we see that helicity and chirality are identified in the collinear frame.

\subsection{The generalized sigma model}

A generalized sigma model contains nucleons and other $I=\frac{1}{2}$ baryons which are sums of any number of $\left(\frac{1}{2}, 0\right)$ and $\left(0, \frac{1}{2}\right)$ representations; that is, models where $c_{m}=d_{n}=0$ in equation $(14 a)$. These models are unrealistic as $g_{A}$ cannot exceed unity. This is easily seen from equation (17):

$$
\left|g_{A}\right|=\left|\frac{\sum_{l}\left|a_{l}\right|^{2}-\sum_{k}\left|b_{k}\right|^{2}}{\sum_{l}\left|a_{l}\right|^{2}+\sum_{k}\left|b_{k}\right|^{2}}\right| \leqslant 1 .
$$

Hence, it is not possible to reach the physical value of $g_{A}$ unless the nucleon couples to at least one state which transforms as $\left(1, \frac{1}{2}\right)$ or $\left(\frac{1}{2}, 1\right)$. Put another way, unless the nucleon couples to $\Delta$, or some other $I=\frac{3}{2}$ state, $g_{A}$ is constrained by the bound in equation (21). 


\subsection{The non-relativistic quark model}

The simplest realistic representation places $N$ and $\Delta$ in an irreducible $\left(\frac{1}{2}, 1\right)$ representation:

$$
\begin{aligned}
& \left|N, \frac{1}{2}\right\rangle=\left|\frac{1}{2}, 1\right\rangle_{\frac{1}{2}}, \\
& \left|\Delta, \frac{1}{2}\right\rangle=\left|\frac{1}{2}, 1\right\rangle_{\frac{3}{2}} .
\end{aligned}
$$

That is, $a_{l}=b_{k}=d_{n}=e_{l}=f_{k}=h_{n}=0$ and $c_{m}=g_{m}=\delta_{m 1}$ in equation (14). This representation is interesting because it gives results equivalent to the spin-flavour $S U(4)$ predictions of the NCQM [23, 28]. For the couplings and masses we find

$$
g_{A}=\frac{5}{3}, \quad \mathcal{C}_{\Delta N}=2, \quad M_{N}^{2}=M_{\Delta}^{2} .
$$

This representation is unrealistic since the $\Delta$ and the nucleon are degenerate. In extensions of the NCQM one effectively perturbs around this basis in order to split the nucleon and the $\Delta$ and quench the axial couplings.

\subsection{A minimal realistic model}

As we have seen above, a more interesting and realistic scenario is one in which $N, \Delta$ and $N^{\prime}$ saturate the Adler-Weisberger sum rule. One can easily show that the unique solution in which these three states communicate by pion emission and absorption is a reducible sum $\left(0, \frac{1}{2}\right) \oplus\left(\frac{1}{2}, 1\right)[19,20]$. For $\lambda=\frac{1}{2}$ we can write this representation in terms of a single mixing angle $\theta$ as

$$
\begin{aligned}
& \left|N, \frac{1}{2}\right\rangle=\sin \theta\left|0, \frac{1}{2}\right\rangle+\cos \theta\left|\frac{1}{2}, 1\right\rangle_{\frac{1}{2}}, \\
& \left|N^{\prime}, \frac{1}{2}\right\rangle=-\cos \theta\left|0, \frac{1}{2}\right\rangle+\sin \theta\left|\frac{1}{2}, 1\right\rangle_{\frac{1}{2}}, \\
& \left|\Delta, \frac{1}{2}\right\rangle=\left|\frac{1}{2}, 1\right\rangle_{\frac{3}{2}} .
\end{aligned}
$$

From equations (24) and (16) we immediately find

$$
\begin{aligned}
& g_{A}=1+\frac{2}{3} \cos ^{2} \theta, \\
& g_{A}^{\prime}=\frac{2}{3} \sin \theta \cos \theta, \\
& g_{A}^{\prime \prime}=1+\frac{2}{3} \sin ^{2} \theta, \\
& \mathcal{C}_{\Delta N}=2 \cos \theta, \\
& \mathcal{C}_{\Delta N^{\prime}}=2 \sin \theta,
\end{aligned}
$$

which clearly is consistent with equation (13), the Adler-Weisberger sum rule for $\pi N$ scattering. It is straightforward to show that there is a single relation involving the three masses and the mixing angle, $\theta$ :

$$
\cos ^{2} \theta M_{N}^{2}+\sin ^{2} \theta M_{N^{\prime}}^{2}=M_{\Delta}^{2} .
$$

The constraint of no inelastic diffraction from equation (20) implies maximal mixing, $\theta=45^{\circ}$, which results in $g_{A}=1.33$ and $M_{N^{\prime}}=1467 \mathrm{MeV}$. Other predictions are shown in table 2. This value of the nucleon axial coupling is consistent with its chiral limit value, as determined by the process $\pi N \rightarrow \pi \pi N$ [29]. We have also given predictions that follow from fitting the mixing angle to $g_{A}=1.26$. The error bars on the experimental values correspond to the PDG ranges for the decay widths and branching fractions and therefore are seriously 
Table 2. Comparison of chiral predictions with experiment. In the second column (TH1) we fit $\theta=51^{\circ}$ from $g_{A}=1.26$, the physical value of the axial coupling. In the third column (TH2) we assume maximal mixing $\theta=45^{\circ}$, consistent with the constraint of no inelastic diffraction.

\begin{tabular}{lllll}
\hline & TH1 & TH2 & EXP & Process \\
\hline$M_{N^{\prime}}$ & 1386 & 1467 & $1440 \pm 30$ & $N \pi \rightarrow N \pi, \ldots$ \\
$g_{A}$ & 1.26 (input) & 1.33 & 1.26 & $N \rightarrow N \pi$ \\
$g_{A}^{\prime}$ & 0.33 & 0.33 & $0.71 \pm 0.20$ & $N^{\prime} \rightarrow N \pi$ \\
$g_{A}^{\prime \prime}$ & 1.41 & 1.33 & - & $N^{\prime} \rightarrow N^{\prime} \pi$ \\
$\mathcal{C}_{\Delta N}$ & 1.25 & 1.41 & $1.51 \pm 0.10$ & $\Delta \rightarrow N \pi$ \\
$\mathcal{C}_{\Delta N^{\prime}}$ & 1.56 & 1.41 & $1.38 \pm 0.50$ & $N^{\prime} \rightarrow \Delta \pi$ \\
\hline
\end{tabular}

underestimated, particularly for the Roper axial couplings. Note that this chiral representation accounts for the Roper mass while quenching the nucleon axial couplings from the NCQM values toward the experimental values. We find this to be compelling evidence that this chiral representation is perturbatively close to nature. Of course, it would be of great interest to identify novel measurements that are best able to confirm or refute the point of view taken in this paper. Since the $N-N^{\prime}$ mass splitting is of order the kaon mass, the chiral (continuum) corrections to this chiral multiplet can be computed using chiral perturbation theory [30].

\subsection{A second reducible model}

A second interesting scenario is one in which $N, \Delta, N^{\prime}$ and $\Delta^{\prime}(\Delta(1600))$ saturate the AdlerWeisberger sum rule. Here there are several ways in which these states can be embedded in the chiral algebra. Here we choose a reducible sum of $\left(1, \frac{1}{2}\right)$ and $\left(\frac{1}{2}, 1\right)$. For $\lambda=\frac{1}{2}$ we can write this representation in terms of two mixing angles, $\phi$ and $\delta$ as

$$
\begin{aligned}
& \left|N, \frac{1}{2}\right\rangle=\sin \phi\left|\frac{1}{2}, 1\right\rangle_{\frac{1}{2}}^{\prime}+\cos \phi\left|1, \frac{1}{2}\right\rangle_{\frac{1}{2}}, \\
& \left|N^{\prime}, \frac{1}{2}\right\rangle=-\cos \phi\left|\frac{1}{2}, 1\right\rangle_{\frac{1}{2}}^{\prime}+\sin \phi\left|1, \frac{1}{2}\right\rangle_{\frac{1}{2}}, \\
& \left|\Delta, \frac{1}{2}\right\rangle=\sin \delta\left|\frac{1}{2}, 1\right\rangle_{\frac{3}{2}}^{\prime}+\cos \delta\left|1, \frac{1}{2}\right\rangle_{\frac{3}{2}}, \\
& \left|\Delta^{\prime}, \frac{1}{2}\right\rangle=-\cos \delta\left|\frac{1}{2}, 1\right\rangle_{\frac{3}{2}}^{\prime}+\sin \delta\left|1, \frac{1}{2}\right\rangle_{\frac{3}{2}} .
\end{aligned}
$$

From equations (27) and (16) we immediately find

$$
\begin{aligned}
& g_{A}=\frac{5}{3} \cos 2 \phi, \\
& g_{A}^{\prime}=\frac{5}{3} \sin 2 \phi, \\
& g_{A}^{\prime \prime}=\frac{5}{3} \cos 2 \phi, \\
& \mathcal{C}_{\Delta N}=\mathcal{C}_{\Delta^{\prime} N^{\prime}}=2 \cos (\phi+\delta), \\
& \mathcal{C}_{\Delta N^{\prime}}=\mathcal{C}_{\Delta^{\prime} N}=2 \sin (\phi+\delta) .
\end{aligned}
$$

It is straightforward to show that there is a single relation that is independent of the mixing angles, and one relation involving the mixing angles:

$$
\begin{aligned}
& M_{N}^{2}+M_{N^{\prime}}^{2}=M_{\Delta}^{2}+M_{\Delta^{\prime}}^{2}, \\
& \left(M_{\Delta^{\prime}}^{2}-M_{\Delta}^{2}\right) \cos 2 \delta=\left(M_{N^{\prime}}^{2}-M_{N}^{2}\right) \cos 2 \phi .
\end{aligned}
$$


With $g_{A}$ as input, equation (28) determines $\phi=21^{\circ}$. Using the masses of $N, \Delta$ and $\Delta^{\prime}$ as input in equation (29a) gives $M_{N^{\prime}}=1790 \mathrm{MeV}$. We then find that equation (29a) has no real solution for $\delta$. We therefore find that this reducible representation is not consistent with the assumed particle content. We will return to this point below.

\section{Discussion}

\subsection{The quark model interpretation}

The spin-flavour structure of the baryon multiplets seems to provide a powerful explanation of why the Adler-Weisberger sum rule is almost completely saturated by the $\Delta$, with smaller contributions from higher states. In the NCQM the nucleon and the $\Delta$ resonance fill out the completely symmetric 20-dimensional representation of spin-flavour $S U(4)$, which we have seen is equivalent to the $\left(\frac{1}{2}, 1\right)$ representation of $S U(2)_{L} \times S U(2)_{R}[23,28]$. In the NCQM the proton and $\Delta^{+}$wavefunctions can be written as

$$
\begin{aligned}
& |p ; \mathbf{2 0}\rangle=\frac{1}{\sqrt{6}}(2|u \uparrow u \uparrow d \downarrow\rangle-|u \uparrow u \downarrow d \uparrow\rangle-|u \downarrow u \uparrow d \uparrow\rangle)+\cdots \\
& \left|\Delta^{+} ; \mathbf{2 0}\right\rangle=\frac{1}{\sqrt{3}}(|u \uparrow u \uparrow d \downarrow\rangle+|u \uparrow u \downarrow d \uparrow\rangle+|u \downarrow u \uparrow d \uparrow\rangle)+\cdots
\end{aligned}
$$

where ... signifies cyclic permutations which are irrelevant for our purpose. The action of the axial-vector operator, $q^{\dagger} \sigma^{3} \tau^{3} q$, on $u \uparrow$ and $d \downarrow$ is +1 and on $u \downarrow$ and $d \uparrow$ is -1 . One then trivially finds $g_{A}=5 / 3$ and $\mathcal{C}_{\Delta N}=2$. Similarly, placing the proton in the completely antisymmetric four-dimensional representation gives rise to the wavefunction

$$
|p ; \mathbf{4}\rangle=\frac{1}{\sqrt{2}}(|u \uparrow u \downarrow d \uparrow\rangle-|u \downarrow u \uparrow d \uparrow\rangle)+\cdots
$$

from which one easily finds $g_{A}=1$. We can then easily recover the axial-coupling predictions from our minimal realistic model by placing $N, N^{\prime}$ and $\Delta$ in a reducible $\mathbf{4} \oplus \mathbf{2 0}$ representation of $S U(4)$ :

$$
\begin{aligned}
& |N\rangle=\sin \theta\left|\mathbf{4} ; 1^{+}\right\rangle+\cos \theta\left|\mathbf{2 0} ; 0^{+}\right\rangle_{4}, \\
& \left|N^{\prime}\right\rangle=-\cos \theta\left|\mathbf{4} ; 1^{+}\right\rangle+\sin \theta\left|\mathbf{2 0} ; 0^{+}\right\rangle_{4}, \\
& |\Delta\rangle=\left|\mathbf{2 0} ; 0^{+}\right\rangle_{16}
\end{aligned}
$$

where the subscripts indicate the spin-flavour content. Here we have included the spatial quantum numbers that one naively expects. Since the $\mathbf{4}$ of spin-flavour $S U$ (4) is completely antisymmetric, it must carry at least one unit of orbital angular momentum. That is, the simplest totally antisymmetric spatial wavefunction has $L=1$, radial quantum number $n=2$ and positive parity in the harmonic oscillator basis. In the NCQM the $\left|\mathbf{4} ; 1^{+}\right\rangle$state is thought to be irrelevant as it requires two quarks in a baryon to be in an excited state. Note that we assumed only that the baryons are in representations of spin-flavour $S U(4)$; we have assumed nothing about dynamics. The presence of orbital angular momentum is quite strange as a nonvanishing nucleon- $\Delta$ mass splitting requires that $\hat{M}_{\bar{q} q}^{2}$, which acts like an order parameter, carry orbital angular momentum. The peculiar NCQM interpretation of the chiral symmetry representations in the collinear frame was noticed long ago by Casher and Susskind [31].

In the NCQM one usually assigns $N^{\prime}$ and $\Delta^{\prime}$ to a radially excited 20-dimensional representation of $S U(4)$. These states then 'mix', through interactions, with the 'ground state' 20-dimensional representation containing $N$ and $\Delta$. But this is precisely the second reducible model that we have analysed above which overpredicts the Roper mass and has no solution for the axial couplings. 


\subsection{The large- $N_{c}$ limit}

Given the peculiar interpretation of the minimal realistic model in the NCQM it is of interest to consider the large- $N_{c}$ limit. For a baryon made of $N_{c}$ (odd) quarks, there are $\left(N_{c}+1\right) \times$ $\left(N_{c}+3\right) / 4$ possible irreducible representations. It can be shown that, for each helicity $\lambda$, the ground state baryons fall into an $\left(\left(N_{c}-2 \lambda\right) / 4,\left(N_{c}+2 \lambda\right) / 4\right)$ irreducible representation of $S U(2)_{L} \times S U(2)_{R}$ [28]. It is then straightforward to find

$$
\begin{aligned}
& g_{A}=\left(N_{c}+2\right) / 3, \\
& \mathcal{C}_{\Delta N}=\sqrt{\left(N_{c}+5\right)\left(N_{c}-1\right)} / 2,
\end{aligned}
$$

which is consistent with the contracted spin-flavour $S U$ (4) symmetry of large- $N_{c}$ QCD [9] and for $N_{c}=3$ is consistent with the $S U$ (4) results.

We may now ask whether our minimal realistic model has a sensible large- $N_{c}$ limit. If we assume that a baryon is made of $N_{c}$ quarks then our model generalizes to $\left(\left(N_{c}-1\right) / 4\right.$, $\left.\left(N_{c}+1\right) / 4\right) \oplus\left(0, \frac{1}{2}\right)$, again with a single mixing angle, $\theta$. Since $M_{\Delta}-M_{N}=O\left(1 / N_{c}\right)$, it follows from equation (26) that $\theta=O\left(1 / N_{c}\right)$. Therefore in the large- $N_{c}$ limit $N^{\prime}$ decouples from the reducible chiral representation and transforms irreducibly in the $\left(0, \frac{1}{2}\right)$ representation (the 4 of $S U(4))$, and $N$ and $\Delta$ transform irreducibly in the $\left(\left(N_{c}-1\right) / 4,\left(N_{c}+1\right) / 4\right)$ representation as required. Hence the minimal realistic representation is consistent with large- $N_{c}$ expectations for the ground-state tower. What is surprising is that data seem to prefer maximal mixing, $\sin \theta \sim \cos \theta$, whereas in the large- $N_{c}$ limit, $\cos \theta \sim 1$ and $\sin \theta \sim 1 / N_{c}$. We do not yet understand what this portends for the $1 / N_{c}$ corrections of the nucleon and $\Delta$ axial couplings. The large- $N_{c}$ scaling of the axial transitions among large- $N_{c}$ towers has been studied using the Adler-Weisberger sum rule in [32].

\section{Conclusion}

The main point to take from this paper is that even at low energies where chiral symmetry is spontaneously broken, there is a sense in which the baryon spectrum falls into reducible representations of the chiral algebra. This has nothing to do with parity doubling near a chiral symmetry restoring phase transition. We have found that existing data suggest that the nucleon and the $\Delta$ and Roper resonances form a reducible sum of $\left(0, \frac{1}{2}\right)$ and $\left(\frac{1}{2}, 1\right)$ representations of the chiral group, with maximal mixing. From the perspective of the naive constituent quark model this is equivalent to placing these states in a reducible $\mathbf{4} \oplus \mathbf{2 0}$ representation of spinflavour $S U$ (4). Our results suggest that other baryons also fall into finite-dimensional chiral representations that in principle can be mapped out at JLab and other experimental facilities. It should be noted, however, that as one moves higher in the excited spectrum the assumption of pole dominance will become increasingly more unreliable due to the broadening of the baryon states. We stress that it is somewhat peculiar that the chiral multiplet involving the nucleon involves only a few states and that the representations enter with approximately equal weight [30]. We find no QCD-based argument which would explain this simple multiplet structure. This is a worthy puzzle whose resolution-we believe-will lead to deep insight into the manner in which the hadron spectrum arises from QCD.

\section{Acknowledgments}

We thank Keh-Fei Liu, Martin Savage and Ishmail Zahed for valuable conversations. This research was supported in part by the DOE grant DE-FG03-00-ER-41132, NSF 
grant No. PHY-0400231 and by DOE contract DE-AC05-84ER40150, under which the Southeastern Universities Research Association (SURA) operates the Thomas Jefferson National Accelerator Facility (SB), and by RIKEN, Brookhaven National Laboratory, the DOE grants DE-AC02-98CH10886, DE-FG03-01ER41196, and DE-FG02-04ER41338, and an Alfred P Sloan Fellowship (UvK).

\section{References}

[1] For a recent review, see Burkert V D 2003 Eur. Phys. J. A 17303

[2] See, for instance, Junkersfeld J (CB-ELSA Collaboration) 2002 Acta Phys. Pol. B 33941

[3] See, for instance, Beck R et al (A2 and TAPS Collaborations) 2002 Acta Phys. Pol. B 33813

[4] See, for instance, Zou B S et al (BES Collaboration) 2002 PiN Newslett. 16174

[5] Sasaki S, Blum T and Ohta S 2002 Phys. Rev. D 65074503

Gockeler M et al (QCDSF Collaboration) 2002 Preprint nucl-th/0206049

Richards D 2001 Nucl. Phys. B 94269

Melnitchouk W et al 2002 Nucl. Phys. 10996

Melnitchouk W et al 2002 Preprint hep-lat/0210042

Lee F X and Leinweber D B 1999 Nucl. Phys. B 73258

Lee F X et al 2002 Nucl. Phys. B $\mathbf{1 0 6} 248$

[6] Lee F X et al 2003 Nucl. Phys. 119296

Sasaki S 2003 Prog. Theor. Phys. Suppl. 151143

Mathur N et al 2005 Phys. Lett. B 605137

[7] Carone C D et al 1994 Phys. Rev. D 505793

Goity J L 1997 Phys. Lett. B 414140

Carlson C E and Carone C D 1998 Phys. Rev. D 58053005

Carlson C E et al 1998 Phys. Lett. B 438327

Carlson C E et al 1999 Phys. Rev. D 59114008

Pirjol D and Yan T-M 1998 Phys. Rev. D 571449

Pirjol D and Yan T-M 1998 Phys. Rev. D 575434

Schat C L, Goity J L and Scoccola N N 2002 Phys. Rev. Lett. 88102002

Schat C L, Goity J L and Scoccola N N 2002 Phys. Rev. D 66114014

[8] Carlson C E and Carone C D 2000 Phys. Lett. B 484260

[9] For a review, see Manohar A V 1998 Preprint hep-ph/9802419

[10] Isgur N and Karl G 1979 Phys. Rev. D 192653

[11] Glozman L Y and Riska D O 1996 Phys. Rep. 268263

[12] Zahed I and Brown G E 1986 Phys. Rep. 1421

[13] Li Z p, Burkert V and Li Z j 1992 Phys. Rev. D 4670

[14] Carlson C E and Mukhopadhyay N C 1991 Phys. Rev. Lett. 673745

[15] Krehl O, Hanhart C, Krewald S and Speth J 2000 Phys. Rev. C 62025207

[16] Cohen T D and Glozman L Y 2002 Phys. Rev. D 65016006

[17] Jido D, Oka M and Hosaka A 2001 Prog. Theor. Phys. 106873

[18] Harari H 1966 Phys. Rev. Lett. 16964

Lipkin H J, Rubinstein H R and Meshkov S 1966 Phys. Rev. 1481405

Gerstein I S and Lee B W 1966 Phys. Rev. Lett. 161060

Harari H 1966 Phys. Rev. Lett. 1756

[19] Gilman F and Harari H 1968 Phys. Rev. 1651803

[20] Weinberg S 1969 Phys. Rev. 1772604

[21] Noga M and Cronström C 1970 Phys. Rev. D 12414

[22] Sudbery A 1970 Nucl. Phys. Phys. B 201 Ogievetskiĭ V I 1971 Sov. J. Nucl. Phys. 13105

[23] Weinberg S 1995 Chiral Dynamics: Theory and Experiment ed A M Bernstein and B R Holstein (Berlin: Springer) Preprint hep-ph/9412326

[24] Zumino B 1969 Theory and Phenomenology in Particle Physics, 1968 International School of Physics 'Ettore Majorana' (New York: Academic Press) p 42

Weinberg S 1970 Lectures on Elementary Particles and Quantum Field Theory ed S Deser et al (Cambridge, MA: MIT Press) p 285 
For a review, see de Alfaro R, Fubini S, Furlan G and Rossetti G 1973 Currents in Hadron Physics (Amsterdam: North-Holland)

[25] Adler S L 1965 Phys. Rev. Lett. 141051

Weisberger W I 1965 Phys. Rev. Lett. 141047

[26] Hagiwara K et al (Particle Data Group) 2002 Phys. Rev. D 66010001

[27] Semenov-Tian-Shanski K, Vereshagin A and Vereshagin V 2003 Preprint hep-ph/0311333

Semenov-Tian-Shanski K, Vereshagin A and Vereshagin V 2003 Preprint hep-ph/0311334

Vereshagin A and Vereshagin V 2004 Phys. Rev. D 69025002

[28] Beane S R 1999 Phys. Rev. D 59031901

[29] Fettes N JUL-3814 (PhD Thesis)

[30] Beane S R and Savage M J 2003 Phys. Lett. B 556142

[31] Casher A and Susskind L 1974 Phys. Rev. D 9436

[32] Broniowski W 1994 Nucl. Phys. A 580429

Wirzba A, Kirchbach M and Riska D O 1994 J. Phys. G. Nucl. Part. Phys. 201583 